\title{
Knockdown of AMPK $\alpha 2$ impairs epithelial-mesenchymal transition in rat renal tubular epithelial cells by downregulating ETS1 and RPS6KA1
}

\author{
XIAOMING YIN, FUJIANG MA, XU FAN, QI ZHAO, XIN LIU and YI YANG
}

Department of Pediatric Urology, Shengjing Hospital of China Medical University, Shenyang, Liaoning 110004, P.R. China

Received April 11, 2020; Accepted August 25, 2020

DOI: $10.3892 / \mathrm{mmr} .2020 .11556$

\begin{abstract}
Epithelial-mesenchymal transition (EMT) serves an important regulatory role in obstructive nephropathy and renal fibrosis. As an intracellular energy sensor, AMP-activated protein kinase (AMPK) is essential in the process of EMT. The aim of the present study was to elucidate changes in the expression levels of AMPK $\alpha 2$ and which AMPK $\alpha 2$ genes play a role during EMT. TGF- $\beta 1$ was used to induce EMT in normal rat renal tubular epithelial (NRK-52E) cells. The short hairpin AMPK $\alpha 2$ lentivirus was used to interfere with AMPK 22 expression levels in EMT-derived NRK-52E cells and AMPK $\alpha 2$ expression levels and EMT were detected. Differential gene expression levels following AMPK $\alpha 2$ knockdown in EMT-derived NRK-52E cells were assessed via gene microarray. Potential regulatory pathways were analyzed using ingenuity pathway analysis (IPA) and differentially expressed genes were partially verified by reverse transcription-quantitative PCR (RT-qPCR) and western blotting. AMPK $\alpha 2$ was upregulated in TGF- $\beta 1$-induced EMT-derived NRK-52E cells. EMT progression was significantly inhibited following downregulation of expression levels of AMPK $\alpha 2$ by shAMPK $\alpha 2$ lentivirus. A total of 1,588 differentially expressed genes were detected following AMPK $\alpha 2$ knockdown in NRK-52E cells in which EMT occurred. The ERK/MAPK pathway was significantly impaired following AMPK $\alpha 2$ knockdown, as indicated by IPA analysis. Furthermore, RT-qPCR and western blot results demonstrated that the expression levels of AMPK $\alpha 2$, v-ets erythroblastosis virus E26 oncogene homolog-1 (ETS1) and ribosomal protein S6 kinase A1 (RPS6KA1) were upregulated following EMT in NRK-52E cells, whereas the expression levels of ETS1 and RPS6KA1 were downregulated following
\end{abstract}

Correspondence to: Professor Yi Yang, Department of Pediatric Urology, Shengjing Hospital of China Medical University, 36 Sanhao, Shenyang, Liaoning 110004, P.R. China

E-mail: yangy2@sj-hospital.org

Key words: epithelial-mesenchymal transition, AMP-activated protein kinase $\alpha 2$, v-ets erythroblastosis virus E26 oncogene homolog-1, ribosomal protein S6 kinase A1, obstructive nephropathy
AMPK $\alpha 2$ knockdown. It was concluded that AMPK $\alpha 2$ plays a key role in the regulation of rat renal tubular EMT, which may be achieved by modulating ETS1 and RPS6KA1 in the ERK/MAPK pathway.

\section{Introduction}

Ureteropelvic junction obstruction is the most common obstructive urinary tract disease in pediatric urology, with an incidence of $0.5-1 / 1,000$ (1). It is also one of the causes of obstructive nephropathy characterized by renal fibrosis (2). The primary features of this irreversible renal fibrosis are glomerular sclerosis and tubulointerstitial fibrosis (3). Previous studies have revealed that tubulointerstitial fibrosis is associated with the process of epithelial-mesenchymal transition (EMT) $(4,5)$. EMT is characterized by a loss of adhesion and polarity of epithelial cells and induction of $\alpha$-smooth muscle actin ( $\alpha$-SMA) $(6,7)$. EMT is involved in numerous pathological changes, including fibrosis and tumor metastasis $(8,9)$. The TGF- $\beta$ signaling pathway serves a key role in regulation of renal fibrosis (10). TGF- $\beta 1$ expression levels are significantly upregulated in the process of renal fibrosis caused by unilateral ureteral obstruction (11).

A number of proteins with differential expression levels between rat kidney tissue from sham operated group and those with complete unilateral ureteral obstruction were identified in our previous study (12). These identified proteins have been reported to be involved in cell apoptosis, energy metabolism and injury of mitochondria and oxidative stress in a preliminary study (12). AMP-activated protein kinase (AMPK), comprising $\alpha 1 / 2, \beta 1 / 2$ and $\gamma 1 / 2 / 3$ subunits, is a mitochondrial energy sensor that detects changes in AMP levels (13). AMPK is involved in the maintenance of cellular energy balance by affecting multiple factors during metabolism (14). For example, activation of AMPK increases the rate of catabolic (ATP-generating) pathways and decreases the rate of anabolic (ATP-utilizing) pathways (15). Increased intracellular ratio of AMP to ATP activates AMPK; AMP binds to the AMPK $\gamma$ subunit, causing conformational changes in the protein and allowing phosphorylation of the Thr-172 site in the $\alpha$ subunit (16). Previous studies have revealed that AMPK, particularly AMPK $\alpha 2$, regulates the EMT process during liver and kidney fibrosis (17-19) and serves an important role in tumor cell metastasis (20). However, the underlying 
mechanisms for AMPK changes in renal tubular EMT remain unclear.

In the present study, the expression levels of $\mathrm{AMPK} \alpha 2$ in EMT-derived normal rat renal tubular epithelial (NRK-52E) cells induced by TGF- $\beta 1$ were investigated. Gene microarray was used to analyze differential gene expression levels in EMT-derived NRK-52E cells before and after AMPK $\alpha 2$ knockdown (KD). Ingenuity pathway analysis (IPA) was performed to identify specific genes and signaling pathways involved in the regulation of EMT by AMPK $\alpha 2$. Finally, reverse transcription-quantitative PCR (RT-qPCR) and western blotting were used to verify the prediction results.

\section{Materials and methods}

Cell culture, RNA interference and TGF- $\beta 1$ EMT induction. NRK-52E cells (Cell Bank of the Chinese Academy of Sciences) were cultured in DMEM (high glucose; cat. no. SH30022.01; Hyclone; Cytiva) supplemented with 10\% FBS (cat. no. SH30071.03; Hyclone; Cytiva) and 3\% penicillin-streptomycin solution (cat. no. SV30010; Hyclone; Cytiva). Cell culture was maintained at $37^{\circ} \mathrm{C}$ in a humidified atmosphere at $5 \% \mathrm{CO}_{2}$. Th cell medium was changed every 2 days.

The sequence of small interfering RNA (5'-GCT GACTTCGGACTCTCTA-3') was designed by Shanghai GeneChem Co., Ltd. for targeting the AMPK $\alpha 2$ sequence (GenBank no. NM_023991). The AMPK $\alpha 2$ hairpin oligonucleotide was inserted into the GV248-GFP lentiviral vector (Shanghai GeneChem Co., Ltd.) to construct a GV248-GFP-short hairpin (sh)AMPK $22 \mathrm{KD}$ vector. The negative control (shCtrl) sequence was 5'-TTCTCCGAA CGTGTCACGT-3', and when incorporated into the lentiviral vector was referred to as GV248-GFP-shCtrl. Then, GV248-GFP-shAMPK $\alpha 2$ KD and GV248-GFP-shCtrl were respectively co-transfected with pHelper 1.0 (Shanghai GeneChem Co., Ltd.) and pHelper 2.0 (Shanghai GeneChem Co., Ltd.) into $293 \mathrm{~T}$ cells (Shanghai GeneChem Co., Ltd.) to package and produce the shRNA expressing and shCtrl lentivirus. The viral titers of AMPK $\alpha 2$ shRNA lentivirus or control lentivirus reached $1 \times 10^{9} \mathrm{TU} / \mathrm{ml}$ for further studies.

NRK-52E cells were seeded in a 6-well tissue culture plate with $1 \times 10^{5} /$ well, 1 day prior to infection. The complete culture solution was replaced by infection enhancing solution with $5 \mu \mathrm{g} / \mathrm{ml}$ polybrene (Shanghai GeneChem Co., Ltd.) and the packed AMPK 22 shRNA lentivirus or control LV was added to the cells with multiplicity of infection 25 . After $12 \mathrm{~h}$, the lentivirus solution was replaced with complete culture solution. At $72 \mathrm{~h}$ post-transfection, the efficacy of AMPK $\alpha 2 \mathrm{KD}$ was validated via RT-qPCR (Fig. S1).

For TGF- $\beta 1$ treatment, all cells were stimulated with $10 \mathrm{ng} / \mathrm{ml}$ recombinant TGF- $\beta 1$ (ProteinTech Group, Inc.) at $37^{\circ} \mathrm{C}$ for $24 \mathrm{~h}$. Cells were divided into four groups: Negative control, TGF- $\beta 1$-treated, TGF- $\beta 1$-treated + shCtrl and TGF- $\beta 1$-treated + shAMPK $\alpha 2$ KD. Protein expression levels and location were determined using immunofluorescence staining. Total RNA was extracted and analyzed using RT-qPCR. Proteins extracted from total cell lysates were analyzed using western blotting. All measurements were repeated $\geq 3$ times.
Gene microarray. The present microarray dataset has been deposited in National Center for Biotechnology Information Gene Expression Omnibus (accession no. GSE141981). The genome-wide effect of AMPK $\alpha 2 \mathrm{KD}$ was assessed using the GeneChip $^{\text {TM }}$ Rat Genome 2302.0 Array (Affymetrix; Thermo Fisher Scientific, Inc.) consisting of 28,000 genes. A total of three biological replicates of EMT-derived NRK-52E cells transduced with shAMPK $\alpha 2$ or shCtrl LV (for $72 \mathrm{~h}$ ) were analyzed using a microarray. RNA was initially isolated using TRIzol ${ }^{\circledR}$ reagent and quality was determined using the NanoDrop 2000 spectrophotometer (NanoDrop; Thermo Fisher Scientific, Inc.) and Agilent Bioanalyzer 2100 (Agilent Technologies, Inc.). Individual microarrays were used for gene expression level profiling of each sample. Briefly, $500 \mathrm{ng}$ RNA samples were reverse-transcribed and labeled with biotin using the GeneChip3' IVT labeling kit according to the manufacturer's protocol. Labeled complementary (c)DNA was then hybridized onto the GeneChip ${ }^{\mathrm{TM}}$ Rat Genome 2302.0 Array. Arrays were performed with the GeneChip ${ }^{\circledR}$ Hybridization, Wash and Stain kit using the GeneChip ${ }^{\circledR}$ Fluidics Station 450. All GeneChip ${ }^{\circledR}$ products were obtained from Affymetrix (Thermo Fisher Scientific, Inc.) and used according to the manufacturer's protocol. The chip array was scanned directly post-hybridization using the GeneChip ${ }^{\circledR}$ Scanner 3000. Microarray data were analyzed using GeneSpring software (version 11; Agilent Technologies, Inc.). P-values were determined using a linear model based on the empirical Bayesian distribution (21). The false discovery rate (FDR) was corrected using the Benjamini-Hochberg method (22). The screening criteria for significantly differential genes were |fold-changel $>3$ and $\mathrm{FDR}<0.05$.

$I P A$. Datasets representing differentially expressed genes derived from microarray analyses were imported into the IPA tool (ingenuity.com; Ingenuity ${ }^{\circledR}$ Systems). The 'core analysis' function in the IPA software was used to interpret differentially expressed data, which included functional signaling pathways. Differentially expressed genes were mapped onto functional signaling pathways available in the Ingenuity database. Z-score activation algorithms were computed using the IPA software. Analyses performed within the IPA program included identification of a particular dataset and its functional signaling pathways.

$R T-q P C R$. Total RNA was isolated from NRK-52E cells using RNAiso Plus (Takara Bio, Inc.) according to the manufacturer's instructions and subjected to reverse transcription into cDNA using the PrimeScript ${ }^{\mathrm{TM}}$ RT Reagent kit (Takara Bio, Inc.) Reverse transcription was performed at $37^{\circ} \mathrm{C}$ for $15 \mathrm{~min}$ and then $95^{\circ} \mathrm{C}$ for $5 \mathrm{sec}$. RT-qPCR analysis was performed using SYBR ${ }^{\circledR}$ Premix Ex Taq ${ }^{\mathrm{TM}}$ (Takara Bio, Inc.) and the ABI ViiA7DX System (Applied Biosystems; Thermo Fisher Scientific, Inc.). $\beta$-actin expression levels were used as an internal reference for all PCR experiments. RT-qPCR primers designed for specific target genes were synthesized by Takara Bio, Inc. (Table SI). PCR reactions were performed using the following cycling conditions: $95^{\circ} \mathrm{C}$ for $30 \mathrm{sec}$, followed by 40 cycles at $95^{\circ} \mathrm{C}$ for $5 \mathrm{sec}, 60^{\circ} \mathrm{C}$ for $30 \mathrm{sec}$ and $72^{\circ} \mathrm{C}$ for $20 \mathrm{sec}$. The relative mRNA levels for each sample were calculated by the $2^{-\Delta \Delta \mathrm{cq}}$ method (23). 
Western blot analysis. Proteins were isolated using an isolation kit (Beyotime Institute of Biotechnology) and quantified using the 2D-Quant kit (Beyotime Institute of Biotechnology) according to the manufacturer's instructions. A total of $50 \mu \mathrm{g}$ protein/lane extracted from cells was separated by $8 \%$ SDS-PAGE and then transferred in Tris- $\mathrm{HCl}$ methanol (20 mM Tris, $150 \mathrm{mM}$ glycine and 20\% methanol) onto PVDF membranes (EMD Millipore) using a Trans-Blot electrophoresis transfer cell (Bio-Rad Laboratories, Inc.). The membranes were subsequently blocked with $5 \%$ non-fat dry milk in TBS containing $0.1 \%$ Tween-20 for $2 \mathrm{~h}$ at room temperature and incubated with primary antibodies overnight at $4^{\circ} \mathrm{C}$. Primary antibodies included AMPK $\alpha 2$ (polyclonal rabbit; 1:2,000; product code ab3760; Abcam), v-ets erythroblastosis virus E26 oncogene homolog-1 (ETS-1) (monoclonal rabbit; 1:1,000; product no. 14069S; Cell Signaling Technology,Inc.), homolog-1 and ribosomal protein s6 kinase A1 (RPS6KA1) (monoclonal rabbit; 1:1,000; product code ab32114; Abcam), E-cadherin (monoclonal mouse; 1:1,000; product no. 14472S; Cell Signaling Technology, Inc.), $\alpha$-SMA (monoclonal mouse; 1:100; product code ab7817; Abcam), vimentin (monoclonal mouse; 1:1,000; product code ab8978; Abcam) and GAPDH (monoclonal rabbit; 1:10,000; product code ab181602; Abcam). Following three washes in TBST (10 $\mathrm{min} /$ wash), the membranes were incubated with goat anti-rabbit or anti-mouse IgG-HRP (both 1:2,000; product codes ab6721 and ab6789, respectively; both Abcam) secondary antibodies for $1 \mathrm{~h}$ at room temperature and were washed again. All immunoblots were performed $\geq 3$ times. The antigen-antibody complexes were visualized using enhanced chemiluminescence reagents (Thermo Fisher Scientific, Inc.). GAPDH was used as a loading control. Detected bands were quantified using ImageJ 2x software (version 2.1.4.7; National Institutes of Health). The relative density of each protein was calculated by dividing the optical density value of each protein by that of the loading control.

Immunofluorescence staining. NRK-52E cells $\left(1 \times 10^{6}\right)$ were washed with PBS and fixed in $4 \%$ paraformaldehyde for $30 \mathrm{~min}$ at room temperature. Fixed cells were washed again with PBS and permeabilized in $0.5 \%$ Triton X-100 diluted in PBS for $10 \mathrm{~min}$ at room temperature. The cells on the slides were subsequently blocked with $5 \%$ bovine serum albumin (Beyotime Institute of Biotechnology) for $1 \mathrm{~h}$ at room temperature. Subsequently, the negative control and TGF- $\beta 1$-treated groups were incubated with the AMPK $\alpha 2$ antibody (polyclonal rabbit; 1:100; product code ab3760; Abcam) at $4^{\circ} \mathrm{C}$ overnight. The TGF- $\beta 1$-treated + shCtrl and TGF- $\beta 1$-treated $+\operatorname{shAMPK} \alpha 2$ $\mathrm{KD}$ groups were incubated with the green fluorescent protein (GFP) antibody (polyclonal rabbit antibody; 1:2,000; product code ab6556; Abcam) at $4^{\circ} \mathrm{C}$ overnight, then incubated with fluorescein isothiocyanate and rhodamine-conjugated goat anti-rabbit (1:100; cat. no. sc-2012; Santa Cruz Biotechnology, Inc.) secondary antibodies. $0.1 \%$ DAPI (BIOSS) was used to stain cell nuclei on glass slides for $5 \mathrm{~min}$ at $37^{\circ} \mathrm{C}$. The cells were then examined using fluorescence microscopy (magnification, x200; Nikon CE1 Confocal Microscope; Nikon Corporation).

Statistical analysis. Data are presented as the mean \pm SEM. The number of repeats was $\geq 3$ times. Statistical significance between two groups was determined using unpaired Student's
A

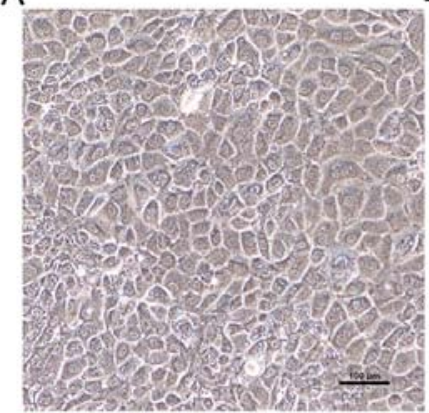

Control
B

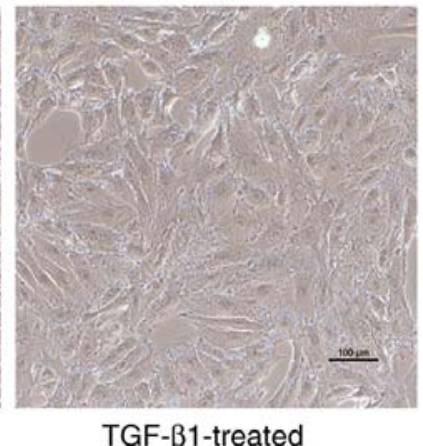

Figure 1. Morphological changes in NRK-52E cells before and after TGF- $\beta 1$ treatment. (A) Morphology of NRK-52E cells. (B) TGF- $\beta 1$ (10 ng/ml) induced morphological changes after $24 \mathrm{~h}$ in NRK-52E cells, which changed from typical round and polygonal to fusiform shape, indicating that the epithelial-mesenchymal transition occurred (magnification, x200).

t-test and between four groups by one-way ANOVA. P-values were calibrated using Bonferroni's correction as a post hoc test. Analysis was performed using SPSS software (version 23.0; IBM Corp.). $\mathrm{P}<0.05$ was considered to indicate a statistically significant difference.

\section{Results}

AMPKa 2 expression levels are upregulated following TGF- $\beta 1$-induced EMT in NRK-52E cells and EMT is impaired in NRK-52E cells following AMPK $\alpha 2$ knockdown. TGF- $\beta 1$ was used to induce EMT in NRK-52E cells. Resulting morphological changes in the NRK-52E cells included a change from typical round and polygonal to fusiform shape, indicating that EMT occurred (Fig. 1). In addition, western blot experiments demonstrated that EMT protein markers $\alpha$-SMA and vimentin were upregulated, whereas epithelial cell protein marker E-cadherin was downregulated, further confirming the occurrence of EMT (Fig. 2).

Immunofluorescence experiments demonstrated that AMPK $\alpha 2$ was primarily expressed in the cytoplasm and nucleus of NRK-52E cells (Fig. 3A). Western blotting and RT-qPCR experiments revealed that AMPK $\alpha 2$ protein and mRNA expression levels were significantly upregulated during EMT in NRK-52E cells (Figs. 3B and 4).

GFP expression levels were observed $72 \mathrm{~h}$ following shAMPK $\alpha 2$ and shCtrl LV transfection in NRK-52E cells that underwent EMT induced by TGF- $\beta 1$, indicating that transfection was successful (Fig. 5A). RT-qPCR demonstrated that the expression levels of AMPK $\alpha 2$ mRNA were downregulated by $70 \%$ in the TGF- $\beta 1$-treated + shAMPK $\alpha 2$ group compared with the TGF- $\beta 1$-treated + shCtrl group, indicating that AMPK $\alpha 2$ was specifically and effectively knocked down (Fig. 5B). In addition, western blot experiments revealed that expression levels of AMPK $\alpha 2$ protein were downregulated in the TGF- $\beta 1$-treated $+\operatorname{shAMPK} \alpha 2$ group compared with the TGF- $\beta 1$-treated + shCtrl group (Fig. 4). The expression levels of $\alpha$-SMA and vimentin were downregulated, whereas E-cadherin expression levels were upregulated, suggesting that the EMT process was inhibited following AMPK 22 knockdown (Fig. 2). These data indicated that AMPK $\alpha 2$ may 

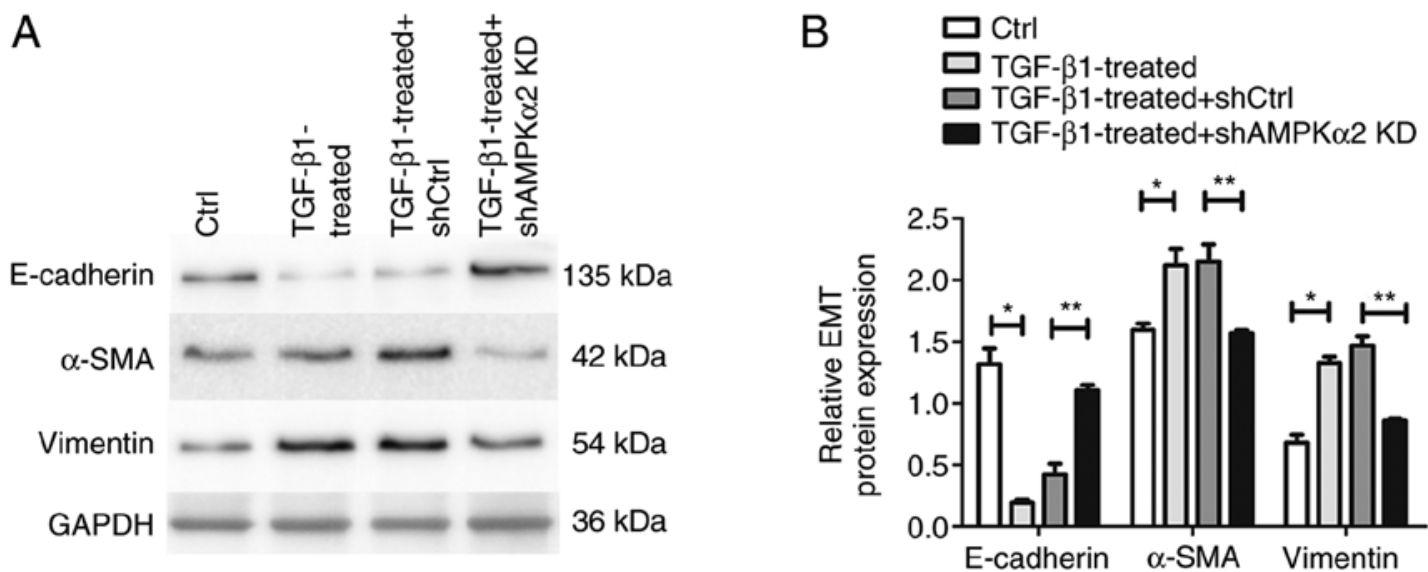

Figure 2. Western blot analysis of EMT-associated proteins $\alpha$-SMA, vimentin and E-cadherin. (A) Western blot images of the EMT-associated proteins $\alpha$-SMA, vimentin, and E-cadherin, representative of $\geq 3$ independent experiments. GAPDH was used as a loading control. (B) Bar graph of western blot analysis. ${ }^{*} \mathrm{P}<0.05$ TGF- $\beta 1$ treated vs. Ctrl; ${ }^{* *} \mathrm{P}<0.05$ TGF- $\beta 1$-treated + shAMPK $\alpha 2$ KD vs. TGF- $\beta 1$-treated + shCtrl. EMT, epithelial-mesenchymal transition; SMA, smooth muscle actin; Ctrl, control; sh, short hairpin; AMPK $\alpha 2$, AMP-activated protein kinase $\alpha 2$; KD, knockdown.

A
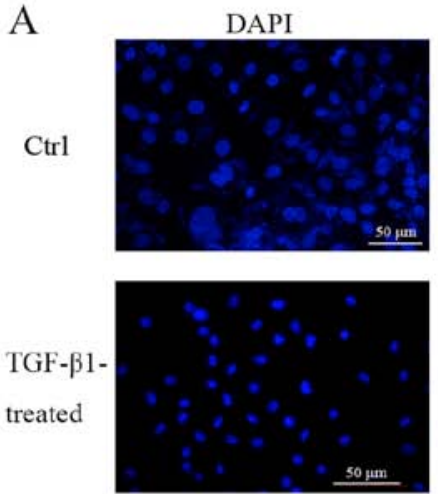
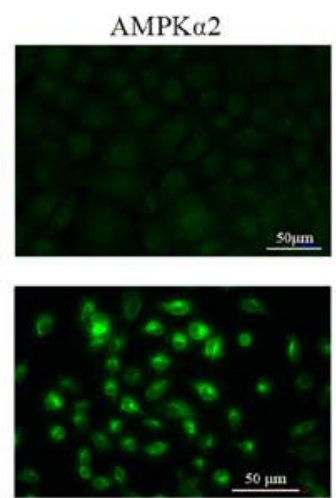
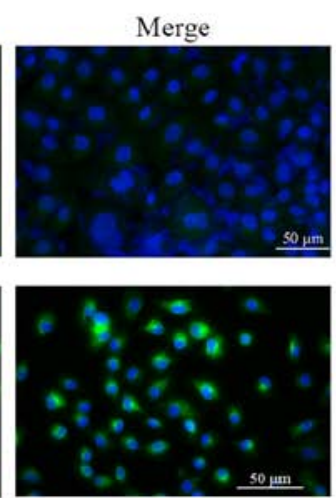

B

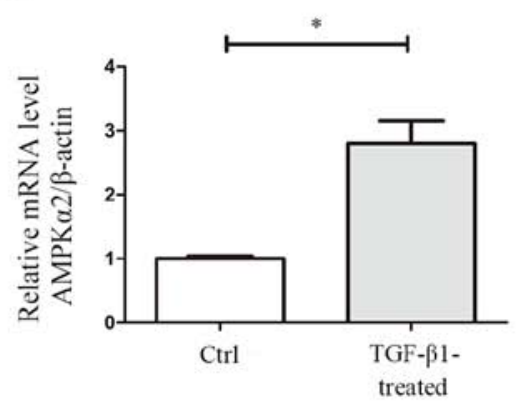

Figure 3. Expression levels of AMPK $\alpha 2$ protein and mRNA detected by immunofluorescence and RT-qPCR. (A) Following TGF- $\beta 1$ treatment, AMPK $\alpha 2$ was primarily expressed in the cytoplasm and nucleus of NRK-52E cells (magnification, x200). (B) Analysis of AMPKa2 mRNA between control- and TGF- $\beta 1$-treated groups by RT-qPCR. $\beta$-actin was used as the endogenous control. ${ }^{*} \mathrm{P}<0.05$ TGF- $\beta 1$-treated vs. Ctrl. AMPK $\alpha 2$, AMP-activated protein kinase $\alpha 2$; RT-q, reverse transcription-quantitative; Ctrl, control.

play a key regulatory role in the process of EMT in NRK-52E cells.

Differential gene expression levels and IPA following AMPKa2 KD in NRK-52E cells with EMT. In order to detect which genes were altered following AMPK $\alpha 2 \mathrm{KD}$, the gene expression level profiles of EMT-derived NRK-52E cells transduced with shAMPK $\alpha 2$ or shCtrl-payload LVs were determined using the GeneChip Rat $2302.0^{\circledR}$ PathArray $^{\mathrm{TM}}$ Rat Gene Expression Array with three biological replicates. A total of 1,588 differentially expressed genes were identified, of which 1,510 were downregulated and 78 were upregulated (Fig. 6A).

Next, IPA was used to perform pathway analysis of these 1,588 differentially expressed genes. The IPA demonstrated that $\mathrm{AMPK} \alpha 2$ may regulate EMT progression in NRK-52E cells via multiple pathways, including ERK/MAPK pathway, Bone morphogenetic protein signaling pathway and Ephrin Receptor Signaling. According to the IPA internal algorithms and standards, a $\mathrm{z}$-score $>2$ represents a significantly activated pathway, whereas $\mathrm{Z}$-score $<-2$ represents a significantly inhibited pathway. In the present study, the ERK/MAPK pathway was significantly inhibited (z-score=-3.550; Fig. 6B). Therefore, AMPK $\alpha 2$ regulation of renal tubular epithelial EMT was achieved via the ERK/MAPK pathway.

IPA results indicated that 36 genes were inhibited in the ERK/MAPK signaling pathway following AMPK 22 knockdown (Table SII; Fig. 6C).

ETS1 and RPS6KA1 in ERK/MAPK signaling pathway are upregulated in NRK-52E cells with EMT, whereas ETS1 and RPS6KA1 are downregulated following AMPK $2 \mathrm{KD}$. A total of five significantly inhibited genes, including HRas proto-oncogene, GTPase (HRAS), CRK proto-oncogene, adaptor protein (CRK), ETS1, RPS6KA1 and cAMP responsive element binding protein 1 (CREB1), were selected for validation. Using RT-qPCR, ETS1 and RPS6KA1 mRNA levels were revealed to be significantly upregulated in the TGF- $\beta 1$-treated group compared with the negative control. The relative mRNA expression levels of ETS1 and RPS6KA1 were significantly downregulated in the TGF- $\beta 1$-treated $+\operatorname{shAMPK} \alpha 2 \mathrm{KD}$ group compared with the TGF- $\beta 1$-treated + shCtrl group (Fig. 7A and B). Inconsistent with microarray results, AMPK $\alpha 2$ downregulation had no effect on HRAS, CRK 


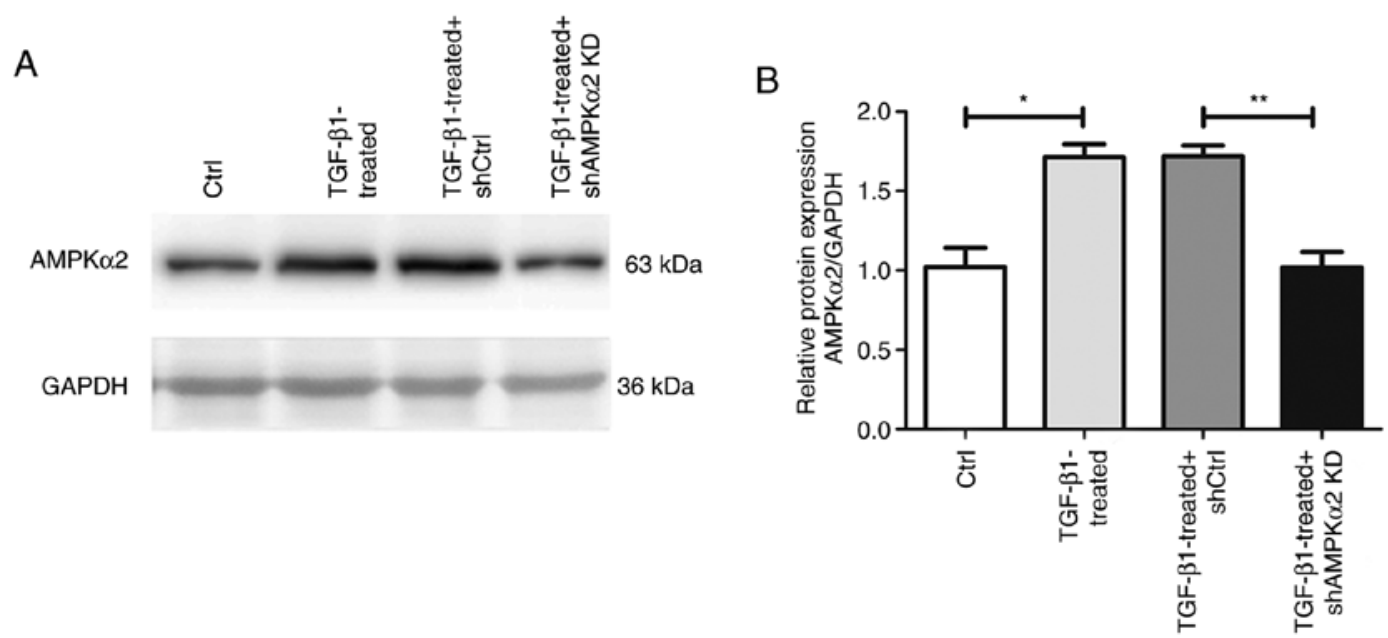

Figure 4. Western blot analysis of AMPK $\alpha 2$. (A) Western blot images of AMPK $\alpha 2$, representative of $\geq 3$ independent experiments. GAPDH was used as a loading control. (B) Bar graph of western blot analysis. " $\mathrm{P}<0.05$ TGF- $\beta 1$-treated vs. Ctrl; ${ }^{* *} \mathrm{P}<0.05$ TGF- $\beta 1$-treated + shAMPK $\alpha 2$ KD vs. TGF- $\beta 1$-treated + shCtrl. AMPK $\alpha 2$, AMP-activated protein kinase $\alpha 2$; Ctrl, control; sh, short hairpin; KD, knockdown.

A
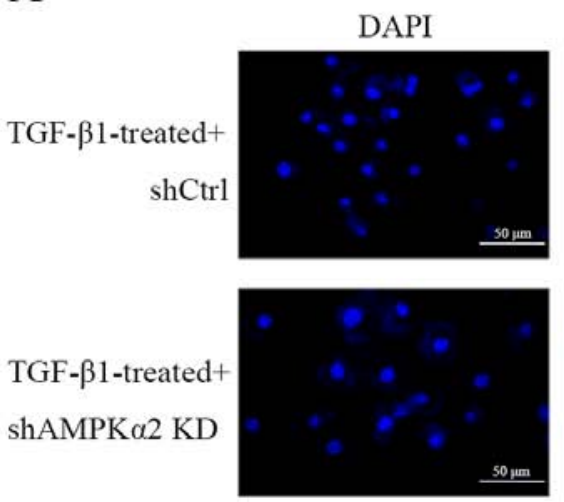
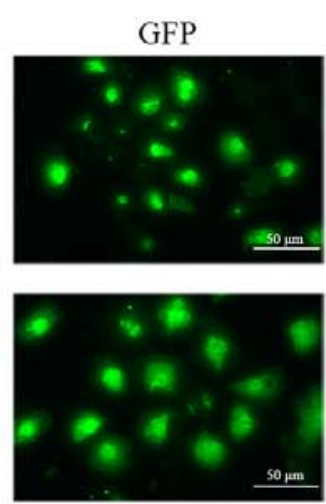
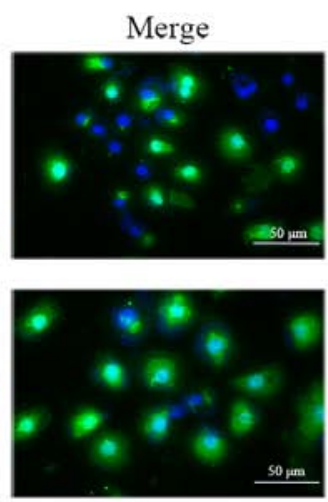

B

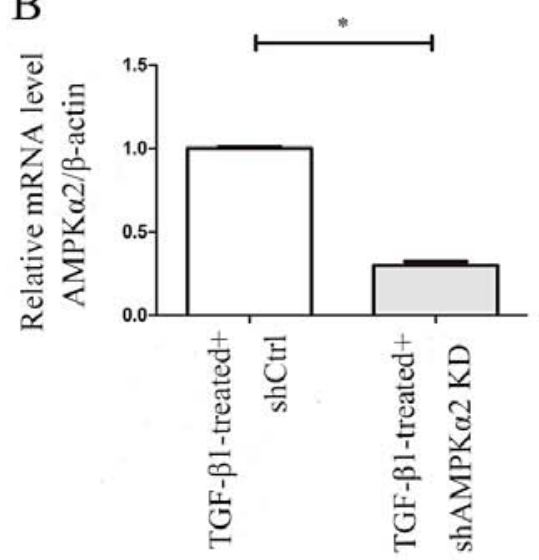

Figure 5. Transfection and KD efficiency of AMPK $\alpha 2$ shRNA and control lentivirus. (A) In EMT-derived NRK-52E cells following $72 \mathrm{~h}$ of transfection with AMPK $\alpha 2$ shRNA and Ctrl lentivirus, GFP expression levels were observed in cells, indicating successful transfection (magnification, x200). (B) AMPK 22 mRNA expression levels in TGF- $\beta 1$-treated + shCtrl and TGF- $\beta 1$-treated + shAMPK $\alpha 2$ KD groups analyzed via reverse transcription-quantitative PCR; housekeeping gene $\beta$-actin was used as the endogenous control. "P<0.05 TGF- $\beta 1$-treated + shAMPK $\alpha 2$ KD vs. TGF- $\beta 1$-treated + shCtrl. KD, knockdown; AMPK $\alpha 2$, AMP-activated protein kinase $\alpha 2$; sh, short hairpin; Ctrl, control; GFP, green fluorescent protein.

and CREB1 (Fig. 7C-E). Western blot experiments revealed that expression levels of ETS1 and RPS6KA1 protein in the TGF- $\beta 1$-treated group were significantly higher than in the negative control. The relative mRNA expression levels of ETS1 and RPS6KA1 were significantly lower in the TGF- $\beta 1$-treated + shAMPK $\alpha 2$ KD group than in the TGF- $\beta 1$-treated + shCtrl group (Fig. 8). Therefore, AMPK $\alpha 2$ may act by regulating ETS1 and RPS6KA1 in the ERK/MAPK signaling pathway during EMT.

\section{Discussion}

Renal fibrosis is considered to be an irreversible process that develops into end-stage renal failure $(24,25)$. EMT serves an important role in obstructive nephropathy and renal fibrosis (26). Therefore, delay, prevention and reversal of renal cell EMT are important for the treatment of obstructive nephropathy (27). In the present study, AMPK $\alpha 2$ served a key role in the EMT of NRK-52E cells and was upregulated in TGF- 31 -induced EMT-derived NRK-52E cells. Interference with AMPK $\alpha 2$ expression levels by shAMPK $\alpha 2$ LV significantly impaired EMT progression. Moreover, using microarray and IPA, it was revealed that AMPK $\alpha 2$ may play an important role in EMT process by regulating ETS1 and RPS6KA1.

AMPK is an AMP-activated protein kinase that detects changes in AMP levels and maintains cell energy balance via multiple pathways, including Hippo-Yes-associated protein pathway and Hedgehog signaling pathway, that affect cellular metabolism, thus serving an important role in kidney disease $(28,29)$. However, current research on promotion or inhibition of EMT following AMPK activation is controversial, which may be due to the biological or cellular specificity of AMPK. The Wang et al (17) study of rat liver cells indicated that AMPK promoted hepatocyte EMT, which leads to liver fibrosis, whereas AMPK-specific inhibitors can limit this process. In addition, EMT also promotes tumor cell 
A

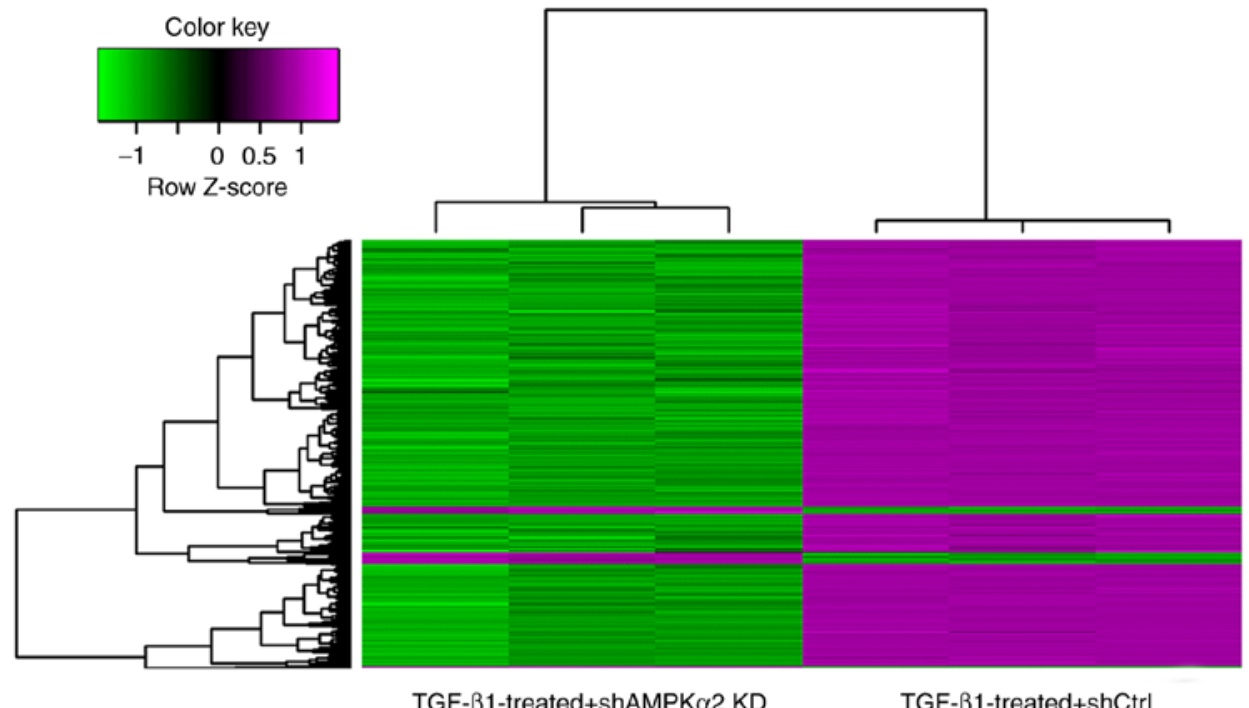

B

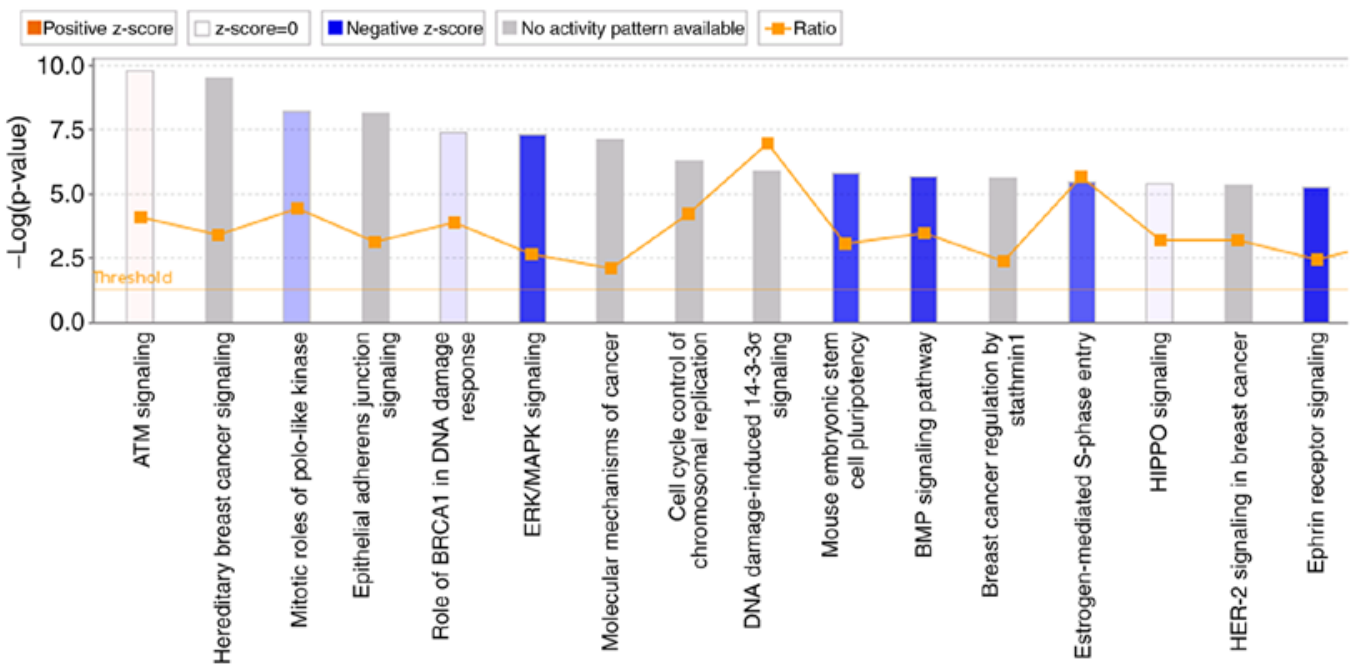

C

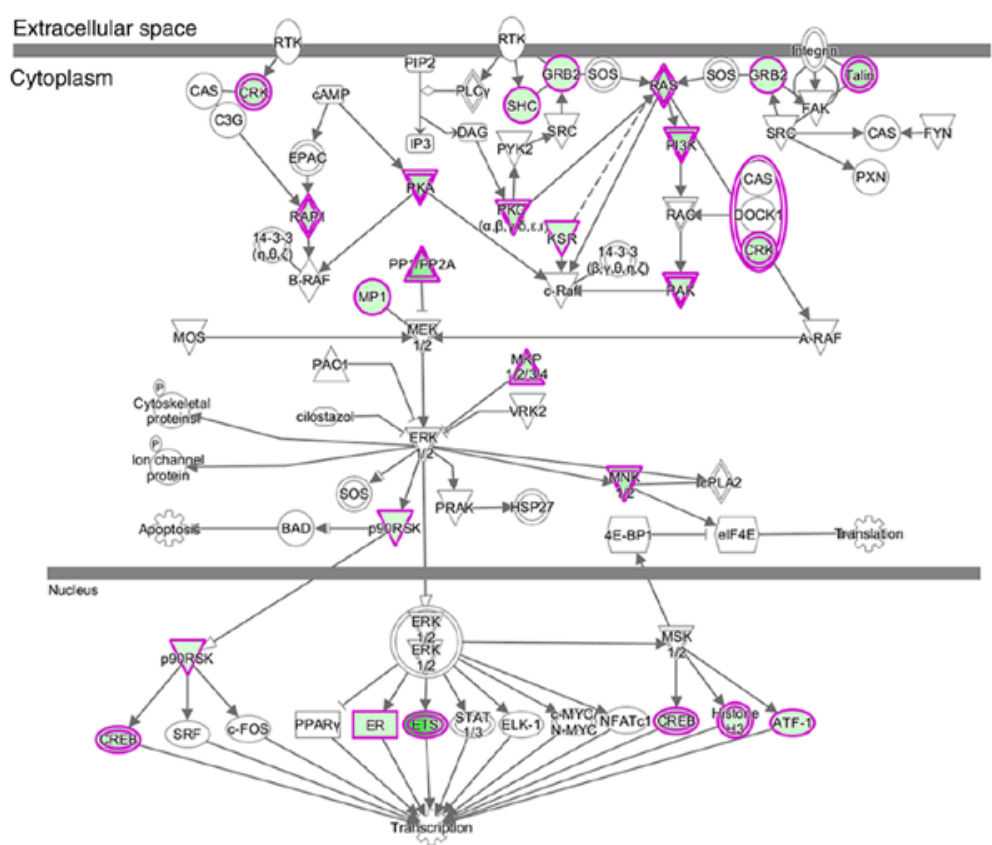

Figure 6. Rat gene chip results and Ingenuity pathway analysis. (A) Differential gene expression level heat map in rat gene chip. (B) Significant enrichment of differential genes in the ingenuity pathway analysis. Orange label indicates pathway activation (z-score $>0$ ), blue label indicates pathway suppression $(\mathrm{z}$-score $<0$ ) and shades of blue (or absolute value of z-score) represent levels of inhibition. ERK/MAPK signaling was significantly inhibited with a z-score of -3.550. (C) ERK/MAPK signaling was significantly inhibited. Purple indicates significant gene upregulation; green indicates significant gene downregulation. sh, short hairpin; AMPK $\alpha 2$, AMP-activated protein kinase $\alpha 2$; KD, knockdown; Ctrl, control. 
A

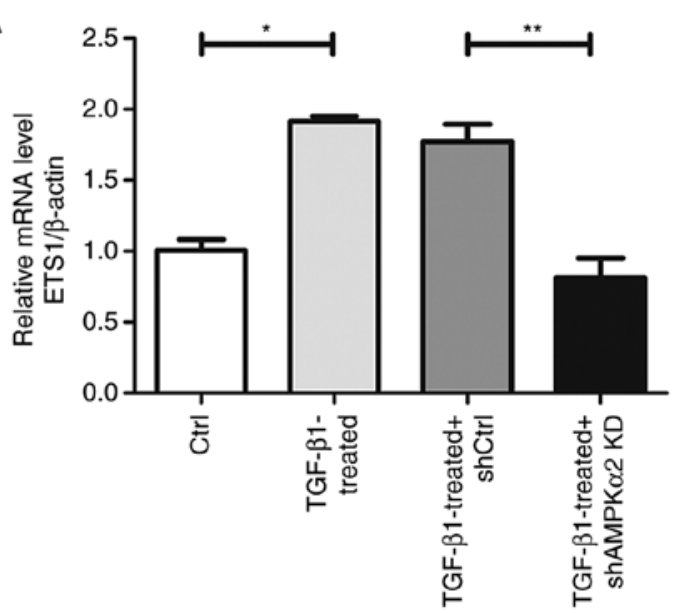

C

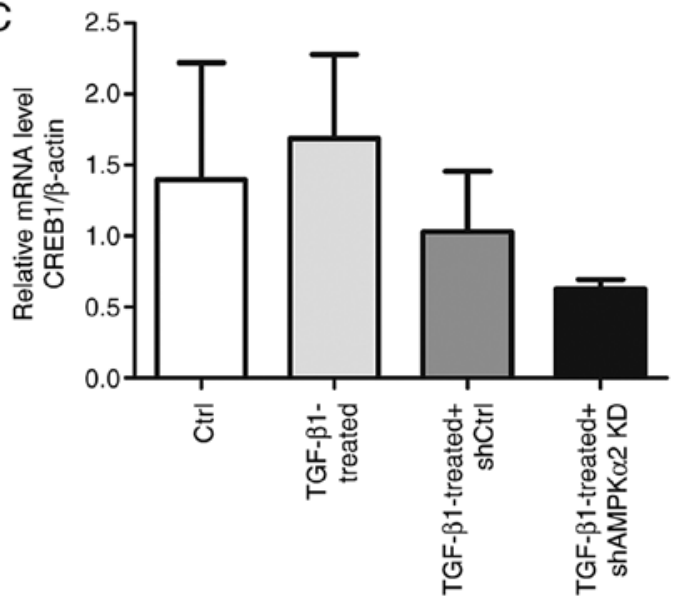

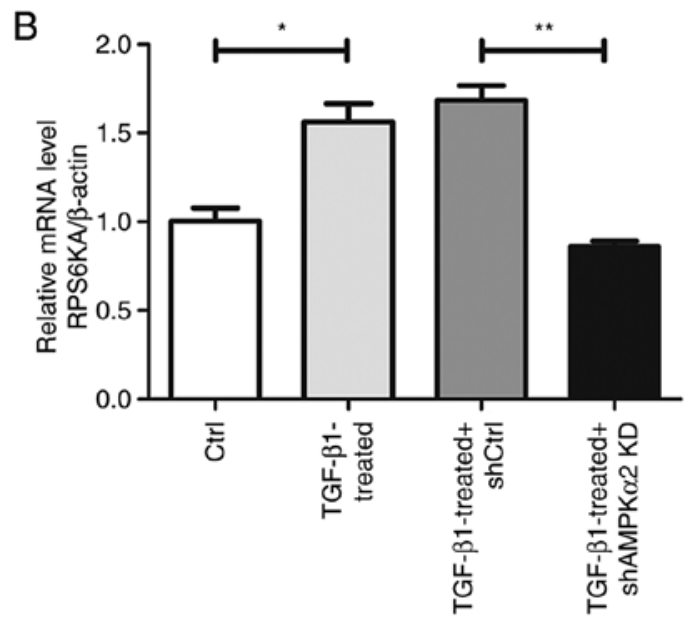

D

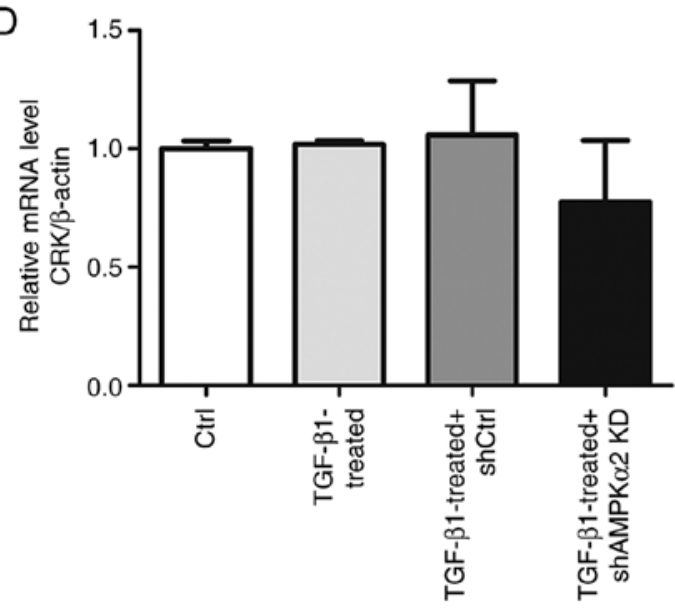

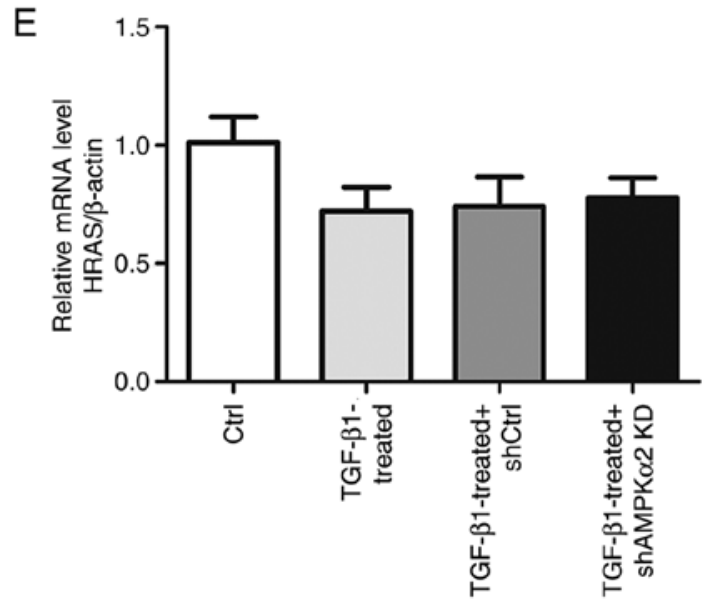

Figure 7. Differentially expressed mRNA levels verified by reverse transcription-quantitative PCR. Relative (A) ETS1, (B) RPS6KA1, (C) CREB1, (D) CRK and (E) HRAS mRNA expression levels. Housekeeping gene $\beta$-actin was used as the endogenous control. "P<0.05 TGF- $\beta 1$-treated vs. Ctrl; ${ }^{* *} \mathrm{P}<0.05$ TGF- $\beta 1$-treated + shAMPK $\alpha 2$ KD vs. TGF- $\beta 1$-treated + shCtrl. ETS1, v-ets erythroblastosis virus E26 oncogene homolog-1; RPS6KA1, ribosomal protein S6 kinase A1; CREB1, cAMP responsive element binding protein 1; CRK, CRK proto-oncogene, adaptor protein; HRAS, HRas proto-oncogene, GTPase; Ctrl, control; sh, short hairpin; AMPK $\alpha 2$, AMP-activated protein kinase $\alpha 2 ; \mathrm{KD}$, knockdown.

metastasis. Studies have shown that in breast and lung cancer, as well as melanoma cells, AMPK activation causes EMT, thereby promoting tumor cell metastasis $(20,30)$. The results of the present study are consistent with these previous findings. However, other studies contradict the hypothesis that AMPK activation increases EMT: Previous research has revealed that activation of AMPK inhibited trans-differentiation of myofibroblasts induced by TGF- $\beta /$ SMAD family member 3 and the occurrence of hepatic astrocytic fibrosis $(31,32)$. In addition, metformin was revealed to activate AMPK to inhibit the 
A

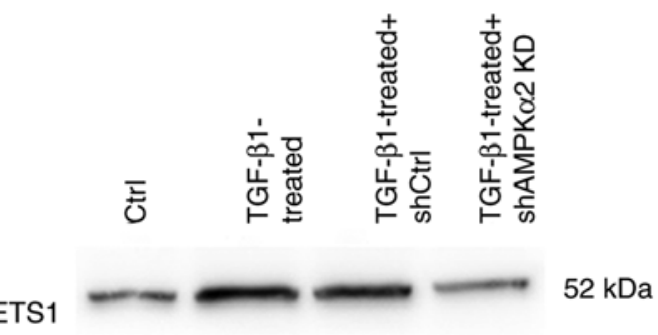

GAPDH

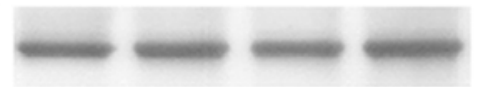

$36 \mathrm{kDa}$
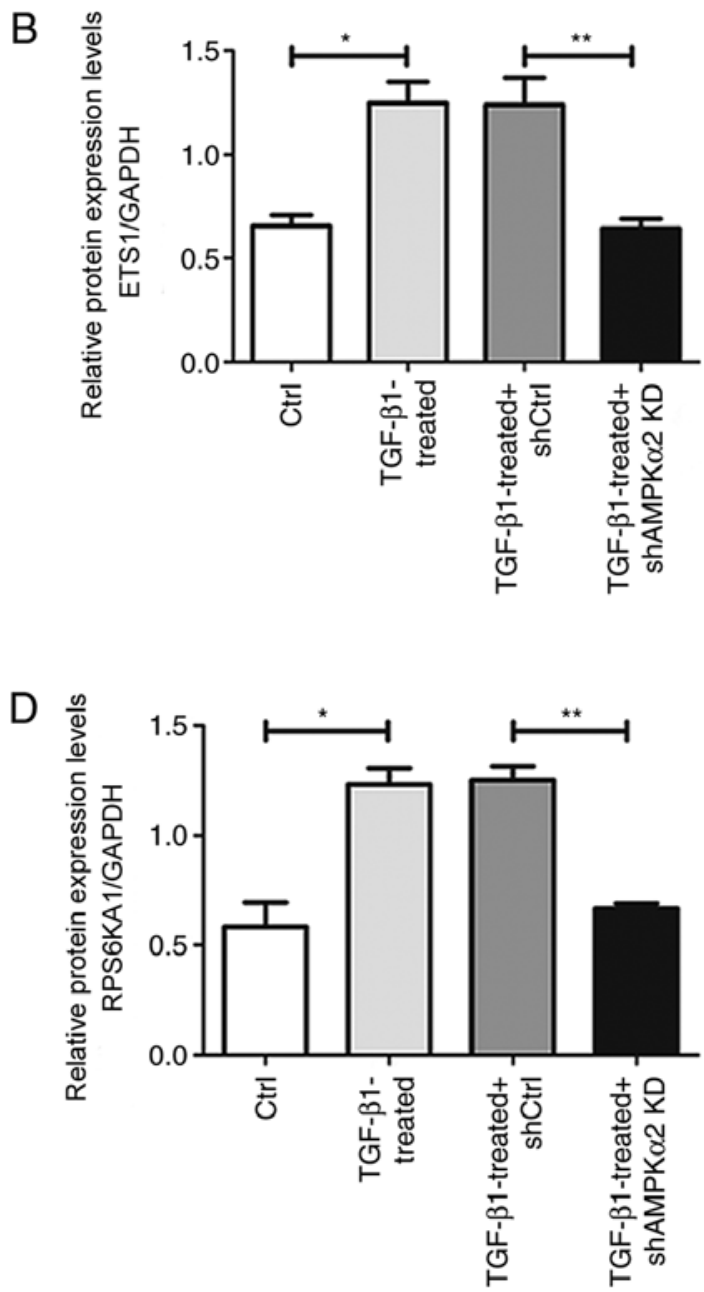

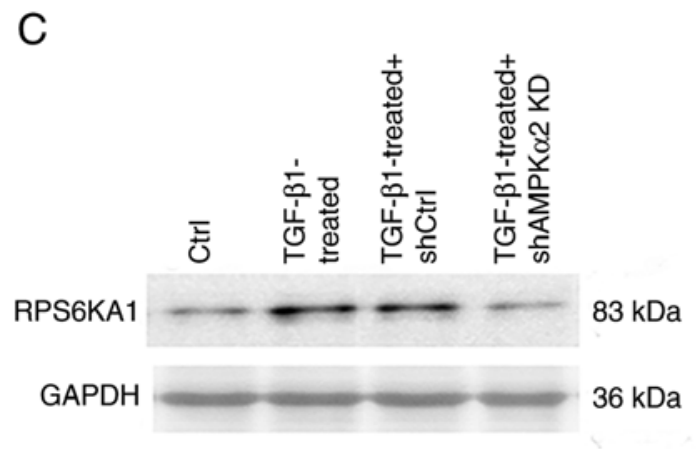

Figure 8. Western blot analysis of ETS1 and RPS6KA1. (A) Western blot images of ETS1, representative of $\geq 3$ independent experiments. GAPDH was used as a loading control. (B) Bar graph of western blot analysis of ETS1. (C) Western blot images of RPS6KA1, representative of $\geq 3$ independent experiments. GAPDH was used as a loading control. (D) Bar graph of western blot analysis of RPS6KA1. "P $<0.05$ TGF- $\beta 1$-treated vs. Ctrl; ${ }^{* *} \mathrm{P}<0.05$ TGF- $\beta 1$-treated + shAMPK $\alpha 2$ KD vs. TGF- $\beta 1$-treated + shCtrl. ETS1, v-ets erythroblastosis virus E26 oncogene homolog-1; RPS6KA1, ribosomal protein S6 kinase A1; Ctrl, control; sh, short hairpin; AMPK $\alpha 2$, AMP-activated protein kinase $\alpha 2$; KD, knockdown.

TGF- $\beta$ signaling pathway and alleviate the EMT process in kidney in a rat model of renal ischemia-reperfusion injury (18). These inconsistent results may be due to tissue specificity or distinct EMT models. In the present study, TGF- $\beta 1$ was used to induce EMT in NRK-52E cells and the expression levels of AMPK $\alpha 2$ were upregulated. EMT was inhibited following LV $\mathrm{AMPK} \alpha 2 \mathrm{KD}$, indicating that AMPK $\alpha 2$ serves an important role in the EMT of NRK-52E cells.

In order to investigate the expression levels of differential genes in EMT-derived NRK-52E cells before and after AMPK $\alpha 2 \mathrm{KD}$, high-throughput analysis was used. According to the gene chip and IPA results, genes in the ERK/MAPK pathway were strongly inhibited following AMPK $\alpha 2 \mathrm{KD}$, indicating that AMPK $\alpha 2$ modulates renal tubular EMT by inhibiting the ERK/MAPK pathway. A total of five genes that were strongly inhibited in the ERK/MAPK pathway, including HRAS, CRK, ETS1, RPS6KA1 and CREB1, were selected for validation. Using RT-qPCR, ETS1 and RPS6KA1 were found to be highly expressed in EMT-derived NRK-52E cells. ETS1 and RPS6KA1 gene expression levels decreased following $\mathrm{AMPK} \alpha 2 \mathrm{KD}$, which is consistent with the microarray results, indicating that AMPK $\alpha 2 \mathrm{KD}$ inhibits EMT by downregulating
ETS1 and RPS6KA1 gene expression levels. However, HRAS, CRK and CREB1 verification results may not be consistent with the microarray results. CRK and CREB1 gene expression levels decreased following AMPK $\alpha 2 \mathrm{KD}$ as shown in microarray and RT-qPCR experiments, but there was no statistical difference in RT-qPCR validation. HRAS gene expression was decreased following $\mathrm{AMPK} \alpha 2 \mathrm{KD}$ in microarray, but increased in RT-qPCR validation.

RPS6KA1 is a member of the serine threonine kinase family and has an $\mathrm{N}$-terminal and a $\mathrm{C}$-terminal kinase domain (33). The C-terminal domain can be activated by ERK1/2 phosphorylation and calcium-dependent kinase (33). Activated RPS6KA1 phosphorylates CREB, NF- $\kappa$ B and other transcription factors (34). RPS6KA1 cause apoptosis of renal tubular epithelial cells during renal fibrosis (35), although to the best of our knowledge, EMT of renal tubular epithelial cells has not yet been reported. ETS1 is a downstream transcription factor of ERK (36). ETS1 is widely expressed in rat kidney and its normal expression levels ensure normal differentiation and development of the kidney (37). A previous study demonstrated that ETS1 may serve a role in the differentiation of liver cells via regulation of the ERK 
pathway (38). ERK/MAPK cascade activation is involved in a number of signaling pathways and comprises a class of important molecules that receive membrane receptor signals and transport them to the nucleus (39). This cascade serves a key role in numerous differentiation-associated signaling pathways, including oxidative stress (39). The present study demonstrated that, in NRK52E cells with EMT, AMPK 22 KD resulted in downregulation of ETS1 and RPS6KA1 in the MAPK/ERK pathway, and the EMT process was impaired. This indicated that AMPK $\alpha 2$ may serve a key role in the EMT of renal tubular epithelial cells by regulating ETS1 and RPS6KA1. AMPK $\alpha 2$ and RPS6KA1 are located in the cytoplasm and nucleus, whereas ETS1 is located in the nucleus. Hypothetically, when oxidative stress occurs, phosphorylation of $\mathrm{AMPK} \alpha 2$ is induced, which may directly or indirectly interact with and activate ETS1 and RPS6KA1. Activated ETS1 and RPS6KA1 may act as transcription factors, regulate the production of EMT-associated proteins, and thereby participate in regulating cell differentiation. The underlying mechanism and association between AMPK $\alpha 2$ and its regulation require further investigation.

The present study had certain limitations. Subsequent experiments are required to verify whether AMPK $\alpha 2$ similarly regulates RPS6KA1 and ETS1 and whether AMPK $\alpha 2$ phosphorylation occurs and how AMPK $\alpha 2$ regulates RPS6KA1 and ETS1 in the human renal tubular EMT. To the best of our knowledge, the present study is the first to demonstrate that AMPK $\alpha 2 \mathrm{KD}$ may impair renal tubular EMT by inhibiting the expression levels of RPS6KA1 and ETS1.

In summary, AMPK $\alpha 2$ serves an important regulatory role in rat renal tubular EMT and this regulation may be achieved by modulating ETS1 and RPS6KA1 in the ERK/MAPK pathway. However, specific AMPK 22 regulation of ETS1 and RPS6KA1 requires further study.

\section{Acknowledgements}

Not applicable.

\section{Funding}

The present study was supported by the National Natural Science Foundation of China (grant. no. 81571514).

\section{Availability of data and materials}

The datasets used and/or analyzed during the current study are available from the corresponding author on reasonable request.

\section{Authors' contributions}

XY and YY conceived and designed the experiments. XY, FM and XF performed the experiments. XY, YY, QZ and XL performed data analysis and wrote the paper. All authors read and approved the final manuscript.

\section{Ethics approval and consent to participate}

Not applicable.

\section{Patient consent for publication}

Not applicable.

\section{Competing interests}

The authors declare that they have no competing interests.

\section{References}

1. Weitz M, Portz S, Laube GF, Meerpohl JJ and Bassler D: Surgery versus non-surgical management for unilateral ureteric-pelvic junction obstruction in newborns and infants less than two years of age. Cochrane Database Syst Rev 7: CD010716, 2016.

2. Klahr S: Obstructive nephropathy. Intern Med 39: 355-361, 2000.

3. Shihab FS: Do we have a pill for renal fibrosis? Clin J Am Soc Nephrol 2: 876-878, 2007.

4. Iwano M: EMT and TGF-beta in renal fibrosis. Front Biosci (Schol Ed) 2: 229-238, 2010.

5. Rastaldi MP: Epithelial-mesenchymal transition and its implications for the development of renal tubulointerstitial fibrosis. J Nephrol 19: 407-412, 2006.

6. Puisieux A, Brabletz T and Caramel J: Oncogenic roles of EMT-inducing transcription factors. Nat Cell Biol 16: 488-494, 2014.

7. Martin-Belmonte F and Perez-Moreno M: Epithelial cell polarity, stem cells and cancer. Nat Rev Cancer 12: 23-38, 2011.

8. Liu Y: Cellular and molecular mechanisms of renal fibrosis. Nat Rev Nephrol 7: 684-696, 2011.

9. Bronsert P, Enderle-Ammour K, Bader M, Timme S, Kuehs M, Csanadi A, Kayser G, Kohler I, Bausch D, Hoeppner J, et al: Cancer cell invasion and EMT marker expression: A three-dimensional study of the human cancer-host interface. J Pathol 234: 410-422, 2014.

10. Kim SI and Choi ME: TGF- $\beta$-activated kinase-1: New insights into the mechanism of TGF- $\beta$ signaling and kidney disease. Kidney Res Clin Pract 31: 94-105, 2012.

11. Zhang D, Sun L, Xian W, Liu F, Ling G, Xiao L, Liu Y, Peng Y, Haruna Y and Kanwar YS: Low-dose paclitaxel ameliorates renal fibrosis in rat UUO model by inhibition of TGF-beta/Smad activity. Lab Invest 90: 436-447, 2010.

12. Zhao Q, Yang Y, Wang CL, Hou Y and Chen H: Screening and identification of the differential proteins in kidney with complete unilateral ureteral obstruction. Int J Clin Exp Pathol 8: 2615-2626, 2015.

13. Hardie DG, Schaffer BE and Brunet A: AMPK: An energy-sensing pathway with multiple inputs and outputs. Trends Cell Biol 26: 190-201, 2016.

14. Jeon SM: Regulation and function of AMPK in physiology and diseases. Exp Mol Med 48: e245, 2016.

15. Carling D: AMPK signalling in health and disease. Curr Opin Cell Biol 45: 31-37, 2017.

16. Hawley SA, Boudeau J, Reid JL, Mustard KJ, Udd L, Mäkelä TP, Alessi DR and Hardie DG: Complexes between the LKB1 tumor suppressor, STRAD alpha/beta and MO25 alpha/beta are upstream kinases in the AMP-activated protein kinase cascade. J Biol 2: 28, 2003.

17. WangX,PanXandSongJ:AMP-activated proteinkinaseisrequired for induction of apoptosis and epithelial-to-mesenchymal transition. Cell Signal 22: 1790-1797, 2010.

18. Wang M, Weng X, Guo J, Chen Z, Jiang G and Liu X: Metformin alleviated EMT and fibrosis after renal ischemia-reperfusion injury in rats. Ren Fail 38: 614-621, 2016.

19. Qiu S, Xiao Z, Piao C, Zhang J, Dong Y, Cui W, Liu X, Zhang Y and $\mathrm{Du} \mathrm{J}$ : AMPK $\alpha 2$ reduces renal epithelial transdifferentiation and inflammation after injury through interaction with CK2 $\beta$. J Pathol 237: 330-342, 2015.

20. Saxena M, Balaji SA, Deshpande N, Ranganathan S, Pillai DM, Hindupur SK and Rangarajan A: AMP-activated protein kinase promotes epithelial-mesenchymal transition in cancer cells through Twist1 upregulation. J Cell Sci 131: cs208314, 2018.

21. Ritchie ME, Phipson B, Wu D, Hu Y, Law CW, Shi W and Smyth GK: limma powers differential expression analyses for RNA-sequencing and microarray studies. Nucleic Acids Res 43: e47, 2015.

22. Benjamini $Y$ and Hochberg Y: Controlling the false discovery rate: A practical and powerful approach to multiple testing. J R Statist Soc B 57: 289-300, 1995. 
23. Livak KJ and Schmittgen TD: Analysis of relative gene expression data using real-time quantitative PCR and the 2(-Delta Delta C(T)) method. Methods 25: 402-408, 2001.

24. Zeisberg M, Maeshima Y, Mosterman B and Kalluri R: Renal fibrosis: Extracellular matrix microenvironment regulates migratory behavior of activated tubular epithelial cells. Am J Pathol 160: 2001-2008, 2002.

25. Sato M, Muragaki Y, Saika S, Roberts AB and Ooshima A: Targeted disruption of TGF-beta1/Smad3 signaling protects against renal tubulointerstitial fibrosis induced by unilateral ureteral obstruction. J Clin Invest 112: 1486-1494, 2003.

26. Stahl PJ and Felsen D: Transforming growth factor-beta, basement membrane, and epithelial-mesenchymal transdifferentiation: Implications for fibrosis in kidney disease. Am J Pathol 159: 1187-1192, 2001.

27. Grande MT and Lopez-Novoa JM: Fibroblast activation and myofibroblast generation in obstructive nephropathy. Nat Rev Nephrol 5: 319-328, 2009.

28. Garcia D and Shaw RJ: AMPK: Mechanisms of cellular energy sensing and restoration of metabolic balance. Mol Cell 66 789-800, 2017.

29. Tain YL and Hsu CN: AMP-activated protein kinase as a reprogramming strategy for hypertension and kidney disease of developmental origin. Int J Mol sci 19: 1744, 2018.

30. He K, Guo X, Liu Y, Li J, Hu Y, Wang D and Song J: TUFM downregulation induces epithelial-mesenchymal transition and invasion in lung cancer cells via a mechanism involving AMPK-GSK3 $\beta$ signaling. Cell Mol Life Sci 73: 2105-2121, 2016.

31. Mishra R, Cool BL, Laderoute KR, Foretz M, Viollet B and Simonson MS: AMP-activated protein kinase inhibits transforming growth factor-beta-induced Smad3-dependent transcription and myofibroblast transdifferentiation. J Biol Chem 283: 10461-10469, 2008.

32. Lim JY, Oh MA, Kim WH, Sohn HY and Park SI: AMP-activated protein kinase inhibits TGF- $\beta$-induced fibrogenic responses of hepatic stellate cells by targeting transcriptional coactivator p300. J Cell Physiol 227: 1081-1089, 2012.
33. Anjum R and Blenis J: The RSK family of kinases: Emerging roles in cellular signalling. Nat Rev Mol Cell Biol 9: 747-758, 2008.

34. Abe JI, Sandhu UG, Hoang NM, Thangam M, QuintanaQuezada RA, Fujiwara K and Le NT: Coordination of cellular localization-dependent effects of sumoylation in regulating cardiovascular and neurological diseases. Adv Exp Med Biol 963: 337-358, 2017.

35. Lin L, Shi C, Sun Z, Le NT, Abe JI and Hu K: The Ser/Thr kinase p90RSK promotes kidney fibrosis by modulating fibroblast-epithelial crosstalk. J Biol Chem 294: 9901-9910, 2019.

36. Plotnik JP, Budka JA, Ferris MW and Hollenhorst PC: ETS1 is a genome-wide effector of RAS/ERK signaling in epithelial cells. Nucleic Acids Res 42: 11928-11940, 2014.

37. Lawrence MC, McGlynn K, Shao C, Duan L, Naziruddin B, Levy MF and Cobb MH: Chromatin-bound mitogen-activated protein kinases transmit dynamic signals in transcription complexes in beta-cells. Proc Natl Acad Sci USA 105: 13315-13320, 2008.

38. Paumelle R, Tulasne D, Kherrouche Z, Plaza S, Leroy C, Reveneau S, Vandenbunder B and Fafeur V: Hepatocyte growth factor/scatter factor activates the ETS1 transcription factor by a RAS-RAF-MEK-ERK signaling pathway. Oncogene 21: 2309-2319, 2002

39. Darling NJ and Cook SJ: The role of MAPK signalling pathways in the response to endoplasmic reticulum stress. Biochim Biophys Acta 1843: 2150-2163, 2014.

This work is licensed under a Creative Commons Attribution-NonCommercial-NoDerivatives 4.0 International (CC BY-NC-ND 4.0) License. 